\title{
Efeito repelente de azadiractina e óleos essenciais sobre Aphis gossypii Glover (Hemiptera: Aphididae) em algodoeiro ${ }^{1}$
}

\author{
Repellent effect of azadirachtin and essential oils on Aphis gossypii Glover \\ (Hemiptera: Aphididae) in cotton plants
}

\author{
Lígia Helena de Andrade ${ }^{2 *}$, José Vargas de Oliveira ${ }^{3}$, Iracilda Maria de Moura Lima ${ }^{4}$, Mauricéa Fidelis de \\ Santana $^{5}$ e Mariana Oliveira Breda
}

\begin{abstract}
RESUMO - A repelência de inseticidas botânicos tem se destacado como uma tática promissora no controle alternativo de pragas agrícolas e urbanas, podendo ser um dos componentes do manejo integrado de pragas. Objetivou-se com este trabalho identificar a repelência de inseticidas botânicos sobre fêmeas ápteras de Aphis gossypii Glover. Testes com chance de escolha foram realizados com discos de folha de algodoeiro, imersos nas caldas dos inseticidas e testemunha (água destilada com DMSO a 2\%). Utilizou-se azadirachtina (0,075\%) e os óleos essenciais de Piper hispidinervum CDC, P. aduncum L., Cymbopogon winterianus (L.), C. citratus (D.C.) Stapf, Foeniculum vulgare Mill, Syzygium aromaticum (L.) Merrill e Perry, Cinnamomum zeylanicum Blume, Schinus terebinthifolius Raddi e Chenopodium ambrosioides L. na concentração de $0,05 \%$. C. citratus, C. winterianus, P. aduncum, S. terebinthifolius, azadirachtina e $C$. zeylanicum apresentaram os maiores percentuais de repelência, 100; 84; 66,67; 64; 60,87 e $48 \%$ respectivamente e reduziram a produção de ninfas em $100 ; 92 ; 42,9 ; 87,5 ; 80,65$ e 89,74\%, apresentando resultados significativos pelo teste do $\chi^{2}$ ao nível de $10 \%$ de probabilidade. Nos testes com $F$. vulgare $\left(\chi^{2}=3,66, \mathrm{P}=0,05\right)$ as fêmeas de $A$. gossypii foram atraídas significativamente para os discos tratados e ocorreu um aumento na produção de ninfas nos resultados obtidos para $F$. vulgare $\left(\chi^{2}=5,87, \mathrm{P}=0,02\right)$ e C. ambrosioides $\left(\chi^{2}=14,31, \mathrm{P}=0,001\right)$.
\end{abstract}

Palavras-chave: Gossypium hirsutum. Pulgão-do-algodoeiro. Comportamento. Inseticidas de origem vegetal.

\begin{abstract}
The repellence of botanical insecticides has emerged as a promising technique in the alternative control of urban and agricultural pests, being seen as one component of integrated pest management. The aim of this work was to identify the repellence of botanical insecticides on apterous females of Aphis gossypii Glover. Random-choice tests were carried out with discs from the leaves of cotton plants immersed in insecticide solution and in a control (distilled water with $2 \%$ DMSO). Azadirachtin was used (0.075\%) and the essential oils of Piper hispidinervum CDC, P. aduncum L., Cymbopogon winterianus (L.), C. citratus (DC) Stapf, Foeniculum vulgare Mill, Syzygium aromaticum (L.) Merrill and Perry, Cinnamomum zeylanicum Blume, Schinus terebinthifolius Raddi and Chenopodium ambrosioides L. at a concentration of $0.05 \%$. C. citratus, C. winterianus, P. aduncum, S. terebinthifolius, azadirachtin and C. zeylanicum showed the highest percentage of repellence, 100, 84, 66.67, 64, 60.87 and 48\% respectively, and reduced nymph production by 100, 92, 42.9, $87.5,80.65$ and $89,74 \%$, with significant results from the $\chi^{2}$ test at $10 \%$ probability. In tests with $F$. vulgare $\left(\chi^{2}=3.66, \mathrm{P}\right.$ $=0.05$ ) females of A. gossypii were significantly attracted to the treated discs, and there was an increase in the production of nymphs in the results obtained for $F$. vulgare $\left(\chi^{2}=5.87, \mathrm{P}=0.02\right)$ and $C$. ambrosioides $\left(\chi^{2}=14.31, \mathrm{P}=0.001\right)$.
\end{abstract}

Key words: Gossypium hirsutum. Cotton aphid. Behaviour. Insecticides of plant origin.

\footnotetext{
*Autor correspondência

'Recebido para publicação em 18/10/2010; aprovado em 03/03/2013

Capítulo de Tese do primeiro autor financiado por bolsa fornecida pela CAPES e CNPq ao mesmo

2Departamento de Ciências Biológicas/UFPI, Br 135, Km 03 s/n, Planalto Horizonte, Bom Jesus-PI, Brasil, 64.900-000, ligia.helenandrade@ gmail.com

${ }^{3}$ Departamento de Agronomia/Entomologia/UFRPE, Av. Dom Manoel de Medeiros s/n, Dois Irmãos, Recife, PE, Brasil, 52.171-900, vargasoliveira@uol.com.br

${ }^{4}$ Departamento de Zoologia/UFAL, P. Afrânio Jorge s/n, Centro, Maceió-Al, Brasil, 57.010-020, iracildalimae @ gmail.com

${ }^{5}$ Departamento de Agronomia/Entomologia, Programa de Pós-Graduação em Entomologia Agrícola/UFRPE, Av. Dom Manoel de Medeiros s/n, Dois Irmãos, Recife-PE, Brasil, 52.171-900, agrocea@yahoo.com.br, breda.mariana@ hotmail.com
} 


\section{INTRODUÇÃO}

A preocupação da sociedade com os problemas decorrentes dos efeitos adversos do uso abusivo de agrotóxicos na agricultura tem despertado o interesse de pesquisas com táticas alternativas de controle de pragas, destacando-se aquelas com os óleos essenciais (FAZOLIN et al., 2005; MARTINEZ; VAN EMDEN, 2001; VENDRAMIM; CASTIGLIONI, 2000).

Esses produtos, provenientes do metabolismo secundário das plantas, são constituídos por misturas complexas de substâncias químicas, como os monoterpenos, sesquiterpenos e flavonóides, que são componentes importantes nos processos de interação planta versus inseto versus inimigo natural, bem como no controle de insetos-praga, ácaros, fungos, bactérias e nematóides (FAZOLIN et al., 2005; OLIVEIRA; VENDRAMIM, 1999; SCHMUTTERER, 1990; TAVARES; VENDRAMIM, 2005).

Atuam nos insetos por ingestão, contato e fumigação e podem ser utilizados como pós, extratos, óleos essenciais e óleos emulsionáveis. (ABRAMSON et al., 2006; ISMAN, 2006; RAJENDRAN; SRIRANJINI, 2008). São geralmente biodegradáveis, de baixa toxicidade para os vertebrados e podem apresentar seletividade para os inimigos naturais (COSME; CARVALHO; MOURA, 2007; SILVA; MARTINEZ, 2004; VIEIRA; MAFEZOLI; BIAVATTI, 2001). Seus efeitos nos insetos incluem mortalidade, reduções na fecundidade, fertilidade, no processo de crescimento, deterrência na alimentação, na oviposição e repelência (MARTINEZ, 2002; ROEL, 2001; ROEL et al., 2000).

Efeitos repelentes de inseticidas de origem vegetal têm sido evidenciados em diversas pragas. Solanum fastigiatum var. acicularium Dunal (jurubeba-do-sul) repeliu o pulgão Brevicoryne brassicae L. (LOVATTO; GOETZE; THOMÉ, 2004), e os óleos essenciais de Illicium verum L. (anis-estrelado) e Cymbopogon citratus (DC.) apresentaram efeito repelente/deterrente (LIMA et al., 2008). Os Óleos essenciais de Lippia origanoides H.B.K., Eucalyptus citriodora Hook. Tagetes lucida Cav. foram repelentes para Sitophilus zeamais Mots. (NERIO; OLIVERO-VERBEL; STASHENKO, 2009), bem como os de Laurus nobilis L., Citrus bergamia Risso, Foeniculum vulgare Mill. e Lavandula hybrida Rev. também repeliram adultos de $S$. zeamais, Cryptolestes ferrugineus (Stephens) e larvas de Tenebrio molitor L. (COSIMI et al., 2009). Nerio, Olivero-Verbel, Stashenko (2010) publicaram revisão recente sobre a atividade de repelência de óleos essenciais sobre insetos, destacando como os mais importantes, os componentes das plantas Cymbopogon spp., Ocimum spp. e Eucaliptus spp.

Devido à falta de segurança oferecida pelo $\operatorname{DEET}(\mathrm{N}$, $\mathrm{N}$-dietil-meta-toluamida e N, N-dietil-3-metilbenzamida), principalmente para crianças, o mercado americano incorporou como repelentes de uso doméstico, os óleos vegetais de citronela, eucalipto e cedro como alternativa a esse produto (ISMAN, 2006).

Assim, visando testar uma tática alternativa de controle que possa ser aproveitada no manejo integrado de pragas do algodoeiro, principalmente em cultivos orgânicos e familiares, objetivou-se com este trabalho avaliar efeitos repelentes de óleos essenciais e azadiractina em fêmeas ápteras de Aphis gossypii Glover.

\section{MATERIAL E MÉTODOS}

Otrabalho foi realizado no Laboratório de Entomologia Agrícola do Departamento de Agronomia (DEPA) da Universidade Federal Rural de Pernambuco (UFRPE), utilizando-se câmaras climatizadas com temperatura e umidade relativa, monitoradas e fotofase de $12 \mathrm{~h}$.

\section{Criação de Aphis gossypii.}

Os insetos foram criados nolaboratóriode Entomologia Agrícola da UFRPE, segundo adaptação da metodologia descrita por Oliveira et al. (2010), sendo mantida em sala climatizada à temperatura de $27 \pm 1^{\circ} \mathrm{C}, 70 \pm 5 \%$ de umidade relativa e fotofase de $12 \mathrm{~h}$.

Sementes de algodoeiro (Gossypium hirsutum L. Raça latifolium Hutch), cultivar CNPA 8H, foram semeadas em bandejas de isopor (272 x $280 \mathrm{~mm}$ e 64 células), contendo substrato Base Plant ${ }^{\circledR}$, constituído de casca de Pinus, vermiculita, turfa, corretivos de acidez e aditivos, com teor de umidade entre 50 e 55\%. Posteriormente, as bandejas foram colocadas dentro de bandejas plásticas, mantendo-se o nível de água adequado para a absorção pelas raízes das plantas, e também contribuir para a manutenção da umidade do ambiente de criação.

Acriaçãofoi iniciada com pulgões coletados em plantas de algodoeiro semeadas em áreas do DEPA. As plantas foram mantidas no interior de gaiolas de germinação e crescimento e de infestação, com as dimensões de 1,0 x 1,20 x 0,60 m, cobertas com tecido "voil". No interior das gaiolas foram instaladas lâmpadas florescentes "luz do dia" e "Grolux" para estimular o processo fotossintético. As bandejas foram colocadas sobre suportes de tubos de PVC, aproximadamente a $60 \mathrm{~cm}$ das lâmpadas. Potes com água e detergente foram mantidos na parte inferior das gaiolas para evitar a infestação de formigas. Os níveis de água das bandejas plásticas e potes foram constantemente observados e completados quando necessário.

As plantas permaneceram por, aproximadamente, 20 diasnas gaiolas de germinaçãoe crescimento, eposteriormente, foram transferidas para as gaiolas de infestação, 
colocando-se folhas com pulgões sobre as mesmas. As colônias foram, periodicamente, observadas para prevenir a presença de parasitóides, predadores e de outros insetos indesejáveis. O processo de criação foi estabelecido, de modo a assegurar o suprimento adequado de plantas e de pulgões para a realização dos experimentos.

\section{Repelência de inseticidas botânicos sobre $A$. gossypii}

Os pulgões foram provenientes da criação estoque do Laboratório de Entomologia Agrícola da UFRPE. As sementes foram semeadas em vasos de 5,0 L com substrato Base Plant $^{\circledR}$ e areia na proporção 1:1 e mantidos em casade-vegetação. Após, aproximadamente, 30 dias, discos de folha de 3,5 cm de diâmetro foram imersos durante $30 \mathrm{~s} \mathrm{em}$ cada concentração dos inseticidas e na testemunha (água destilada) e secos por 30 min à temperatura ambiente. Em seguida, os discos foram colocados, dois a dois (tratado e testemunha) em placas petri de plástico, contendo solução ágar-água a $1 \%$, separados por uma tira de papel ofício com dimensões de 1,3 x 2,0 cm, com tampas contendo abertura de $3,5 \mathrm{~cm}$ de diâmetro no centro, cobertas com tecido tipo "voil". No retângulo de papel foram colocadas fêmeas de A. gossypii de tamanho uniforme. As placas foram vedadas lateralmente com parafilme e mantidas em sala climatizada à temperatura de $24,9 \pm 1,2^{\circ} \mathrm{C}$, umidade relativa de $71,7 \pm 2,8 \%$ e fotofase de $12 \mathrm{~h}$. Decorridas $48 \mathrm{~h}$ foi registrado em cada disco, o número de fêmeas atraídas e de ninfas depositadas.

Os experimentos foram conduzidos no delineamento inteiramente casualizado com cinco repetições, constituídos por oito fêmeas ápteras para os óleos essenciais de Piper hispidinervum CDC, P. aduncum L., Cymbopogon winterianus (L.) e dez fêmeas para C. citratus (D.C.) Stapf, Foeniculum vulgare Mill, Chenopodium ambrosioides L., Syzygium aromaticum (L.) Merrill e Perry, Schinus terebinthifolius Raddi, Cinnamomum zeylanicum Blume e azadirachtina. Os óleos essências foram usados na concentração de $0,05 \%$ em água destilada e DMSO a 2\%, e azadirachtina a 0,075\% em água destilada. Os discos de folha do tratamento testemunha foram imersos em água destilada e DMSO a 2\% (Tabela 1).

Os resultados referentes ao número de pulgões atraídos e número de ninfas produzidas foram analisados pelo teste não-paramétrico $\chi^{2}$ através do programa

Tabela 1 - Inseticidas de origem vegetal testados na avaliação da repelência em Aphis gossypii, em algodoeiro

\begin{tabular}{|c|c|c|c|}
\hline Nome comum & Nome científico & Produto Utilizado & Procedência \\
\hline Nim & Azadirachta indica & Azadirachtina a $1 \%$ & $\begin{array}{l}\text { Quinabra e Trifolio-M GmbH., Química } \\
\text { Natural Brasileira Ltda. SP. }\end{array}$ \\
\hline Pimenta longa & Piper hispidinervum & Óleo essencial & Embrapa - Acre \\
\hline Pimenta-de-macaco & Piper aduncum & Óleo essencial & Embrapa - Acre \\
\hline Citronela & Cymbopogon winterianus & Óleo essencial & $\begin{array}{l}\text { Universidade Federal da Paraíba, Campus } \\
\text { III, Bananeiras, PB. }\end{array}$ \\
\hline Erva-doce & Foeniculum vulgare & Óleo essencial & $\begin{array}{l}\text { Universidade Federal da Paraíba, Campus } \\
\text { III, Bananeiras, PB. }\end{array}$ \\
\hline Erva-de-santa-Maria & Chenopodium ambrosioides & Óleo essencial & $\begin{array}{l}\text { Campos da UFRPE, Laboratório deProdutos } \\
\text { Naturais Bioativos do Departamento de } \\
\text { Química. Recife/PE }\end{array}$ \\
\hline Capim-santo & Cymbopogom citratus & Óleo essencial & $\begin{array}{l}\text { Campos daUFRPE, Laboratório de Produtos } \\
\text { Naturais Bioativos do Departamento de } \\
\text { Química. Recife/PE }\end{array}$ \\
\hline Aroeira & Schinus terebinthifolius & Óleo essencial & $\begin{array}{l}\text { Campos daUFRPE, Laboratóriode Produtos } \\
\text { Naturais Bioativos do Departamento de } \\
\text { Química. Recife/PE }\end{array}$ \\
\hline Cravo-da-índia & Syzygium aromaticum & Óleo essencial & $\begin{array}{l}\text { Campos da UFRPE, LaboratóriodeProdutos } \\
\text { Naturais Bioativos do Departamento de } \\
\text { Química. Recife/PE }\end{array}$ \\
\hline Canela & Cinnamomum zeylanicum & Óleo essencial & $\begin{array}{l}\text { Campos da UFRPE, Laboratório de Produtos } \\
\text { Naturais Bioativos do Departamento de } \\
\text { Química. Recife/PE }\end{array}$ \\
\hline
\end{tabular}


estatístico SAS, comparados pela probabilidade de erro de 5\% (SAS INSTITUTE, 2001). O percentual médio de repelência foi calculado pela fórmula:

$(\mathrm{RP})=[(\mathrm{NC}-\mathrm{NT}) /(\mathrm{NC}+\mathrm{NT}) \times 100]$

sendo: $\mathrm{PR}=$ percentual médio de repelência, $\mathrm{NC}=$ média de insetos na testemunha e $\mathrm{NT}=$ média de insetos no tratamento (OBENG-OFORI, 1995). O percentual de redução de ninfas foi calculado também pela equação 1 , substituindo média de insetos por média de ninfas na testemunha e no tratamento.

\section{RESULTADOS E DISCUSSÃO}

Discos de folhas de algodoeiro tratadas com os óleos essenciais de $C$. citratus $\left(\chi^{2}=33,33, \mathrm{P}^{*}<0,001\right), C$. winterianus $\left(\chi^{2}=6,97, \mathrm{P}^{*}=0,01\right), P$. aduncum $\left(\chi^{2}=6,00\right.$, $\left.\mathrm{P}^{*}=0,01\right)$, S. terebinthifolius $\left(\chi^{2}=11,40, \mathrm{P}^{*}<0,001\right), A$. indica $\left(\chi^{2}=9,39, \mathrm{P}^{*}=0,002\right)$ e $S$. zeylanicum $\left(\chi^{2}=61,12\right.$, $\left.\mathrm{P}^{*}=0,01\right)$, atraíram um menor número de fêmeas ápteras de A. gossypii, em relação à testemunha, sendo, portanto, considerados repelentes. Os óleos de $P$. hispidinervum $\left(\chi^{2}=\right.$ $\left.0,03, \mathrm{P}^{\mathrm{ns}}=0,86\right)$ e de $C$. ambrosioides $\left(\chi^{2}=0,04, \mathrm{P}^{*}=0,84\right)$ não obtiveram resultados significativos de repelência, enquanto o óleo de $F$. vulgare $\left(\chi^{2}=3,66, \mathrm{P}^{*}=0,05\right)$ atraiu um maior número de fêmeas do pulgão quando comparado com a testemunha (Figura 1).

De um modo geral, os óleos que apresentaram repelência, também, diminuíram a produção de ninfas
(Figura 2), sendo os óleos de C. citratus (100\%), C. winterianus $(92,0 \%)$, C. zeylanicus $(89,74 \%), \mathrm{S}$. terebinthifolius $(87,5 \%)$ e azadirachtina $(80,65 \%)$ os que apresentam maior percentual de redução (Tabela 2). Porém, ocorreu um aumento na produção de ninfas nos resultados obtidos para $F$. vulgare $\left(\chi^{2}=5,87, \mathrm{P}^{*}=0,02\right) \mathrm{e}$ C. ambrosioides $\left(\chi^{2}=14,31, \mathrm{P}^{*}=0,001\right)$ (Figura 2).

De acordo com Isman (2006), os óleos essenciais podem atuar de várias maneiras nos insetos, como por contato, ingestão, fumigação, atraentes, repelentes, deterrentes de alimentação e oviposição e nas enzimas digestivas e neurológicas.

$\mathrm{O}$ efeito repelente significativo de algumas plantas tem sido apontado como uma forma muito eficiente em evitar a infestação de pragas em áreas agrícolas, reduzindo a postura e injúrias, e consequentemente, as perdas na produtividade, com benefícios econômicos para os agricultores. Segundo Wäckers; Van Rijn e Bruin (2007), os compostos constitutivos de plantas, os voláteis de flores e os nectários extra-florais também podem exercer influência sobre a busca hospedeira, indicando a presença de recursos nutricionais importantes para o incremento da longevidade e do potencial reprodutivo de insetos.

Alguns estudos semelhantes aos desenvolvidos no presente trabalho constataram resultados positivos sobre repelência de inseticidas de origem vegetal em diferentes pragas.

Assim, Lima et al. (2008) constataram efeito repelente do óleo essencial de Illicium verum L. $(0,05 ; 0,10$ e 0,50\%) e

Figura 1 - Teste com chance de escolha para fêmeas ápteras de A. gossypii $(\mathrm{n}=50)$ atraídas para discos de folha de algodoeiro, não tratados e tratados com inseticidas botânicos na concentração de $0,75 \%$ para Azadirachtina e $0,05 \%$ para os óleos essenciais. Significância (P) através do teste de $\chi^{2}$, sendo resultados significativos ao nível de $5 \%$ (*) e para resultados não significativos (ns). Temperatura de $24,9 \pm 1,2^{\circ} \mathrm{C}$, umidade relativa de $71,7 \pm 2,8 \%$ e fotofase de $12 \mathrm{~h}$

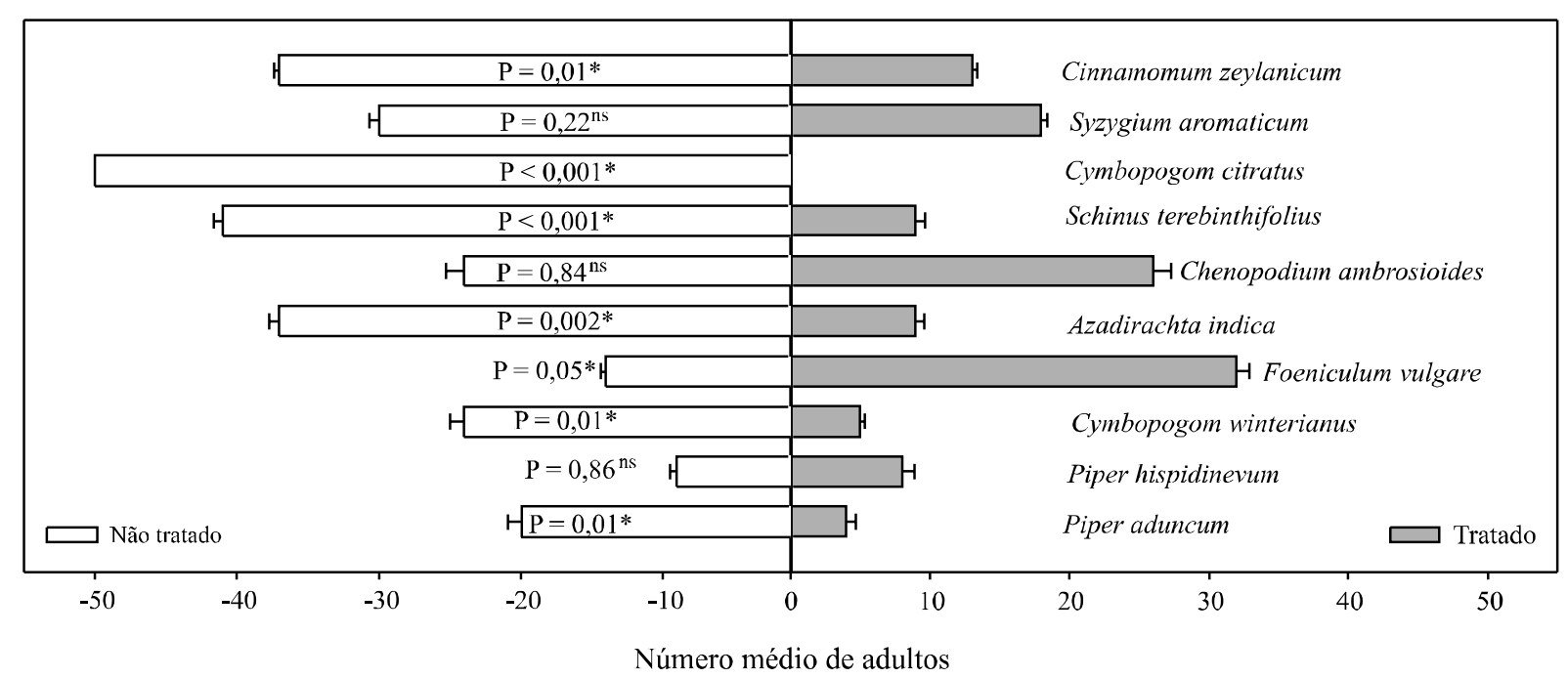


Figura 2 - Ninfas produzidas por fêmeas ápteras de A. gossypii atraídas para discos de folha de algodoeiro não tratados e tratados com inseticidas botânicos, na concentração de $0,075 \%$ para azadiractina e $0,05 \%$ para os óleos essenciais. Significância $(\mathrm{P})$ através do teste de $\chi^{2}$, sendo resultados significativos ao nível de $5 \%(*)$ e para resultados não significativos (ns). Temperatura de $24,9 \pm 1,2{ }^{\circ} \mathrm{C}$, umidade relativa de $71,7 \pm 2,8 \%$ e fotofase de $12 \mathrm{~h}$

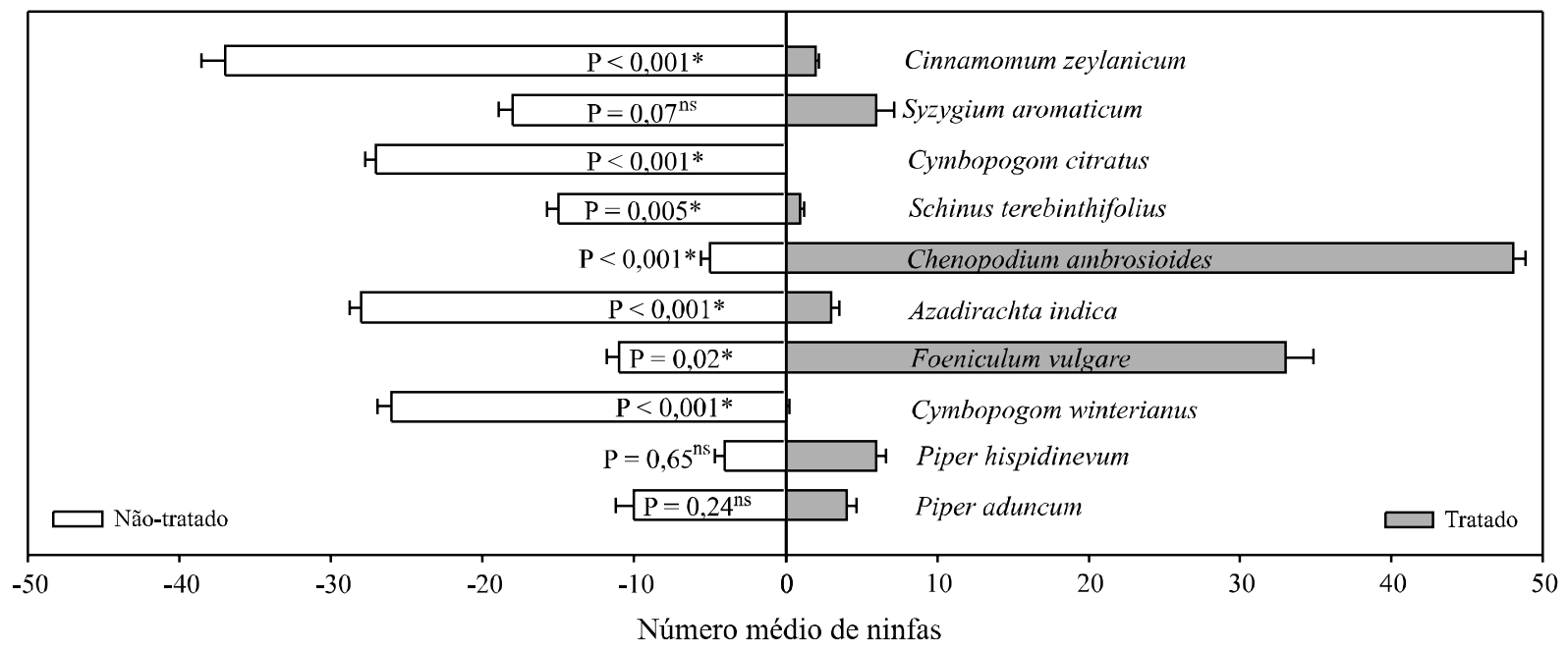

Tabela 2 - Porcentagem de repelência (PR) de adultos e de redução de ninfas de Aphis gossypii mantidos em discos de folha de algodoeiro tratados e não-tradados com inseticidas botânicos, após 48 horas. Temperatura de $24,9 \pm 1,2^{\circ} \mathrm{C}$, umidade relativa de $71,7 \pm 2,8 \%$ e fotofase de $12 \mathrm{~h}$

\begin{tabular}{lccc}
\hline \multicolumn{1}{c}{ Tratamento } & Concentração $(\%)$ & PR $^{1}$ & ${\text { Redução de ninfas }(\%)^{2}}$ \\
\hline Piper hispidinervum & 0,05 & 5,88 & 0 \\
P. aduncum & 0,05 & 66,67 & 42,86 \\
Cymbopogon winterianus & 0,05 & 84,00 & 92,00 \\
Foeniculun vulgare & 0,05 & -3 & -3 \\
Azadirachta indica & 0,075 & 60,87 & 80,65 \\
Chenopodium ambrosioides & 0,05 & -3 & -3 \\
Schinus terebinthifolius & 0,05 & 64 & 87,50 \\
Cymbopogom citratus & 0,05 & 100 & 100,00 \\
Syzygium aromaticum & 0,05 & 25 & 50,00 \\
Cinnamomum zeylanicum & 0,05 & 48 & 89,74 \\
\hline
\end{tabular}

${ }^{1} \mathrm{PR}$ - Porcentagem de repelência de adultos $=[(\mathrm{NC}-\mathrm{NT}) /(\mathrm{NC}+\mathrm{NT}) \times 100]$, sendo $\mathrm{NC}=$ média de adultos na testemunha e NT $=$ média de adultos no tratamento; ${ }^{2} \mathrm{PR}-$ Porcentagem de redução de ninfas $=[(\mathrm{NC}-\mathrm{NT}) /(\mathrm{NC}+\mathrm{NT}) \times 100]$, sendo NC $=$ média ninfas na testemunha e NT $=$ média ninfas no tratamento; ${ }^{3} \mathrm{Não}$ se ajustou à fórmula

C. citratus $(0,01 ; 0,050$ e $0,10 \%)$ em B. brassicae. Lovatto, Goetze e Stashenko (2004), em experimentos de múltipla escolha com folhas de couve, observaram que Solanum fastigiatum var. acicularium Dunal e Solanun diflorum Vell repeliram B. brassicae nas concentrações de 2,5 e 5,0\%.M. persicae foi repelido pelo extrato metanólico de Impatiens parviflora L, aplicado em folhas de tomate (PAVELA; VRCHOTOVÁ; BOZERA, 2009). O efeito repelente associado ao de deterrência alimentar de azadiractina, nos insetos-praga Schistocerca gregaria (Forskal) e Locusta migratoria (Linnaeus) (Orthoptera: Acrididae), Manduca sexta (L.) (Lepidoptera: Sphingidae) e Peridroma saucia (Hübner) (Lepidoptera: Noctuidae) foi mencionado na revisão de Mordue e Blackwell (1993).

Segundo Brito et al. (2006), o inseticida botânico à base de nim, Neemseto ${ }^{\circledR}$, nas concentrações de 0,$25 ; 0,50$ e $1,0 \%$ foi considerado repelente para adultos 
de Tetranychus urticae Koch, e o óleo de nim (Nim-I-Go ${ }^{\circledR}$ ) na concentração de 1,5\% repeliu Brevipalpus phoenicis (Geijskes) (JUSTINIANO et al., 2009).

Os resultados obtidos no presente trabalho indicam que $F$. vulgare apresentou atratividade significativa e os óleos de $C$. citratus, $P$. aduncum, $C$. winterianus e azadirachtina foram repelentes e reduziram a produção de ninfas de $A$. gossypii, evidenciando que são promissores para utilização no manejo integrado desta praga, principalmente em cultivos orgânicos e familiares de algodoeiro. No entanto, estudos adicionais de casa-de-vegetação e campo devem ser conduzidos, visando avaliar os efeitos letais e subletais sobre os inimigos naturais dessa praga.

\section{CONCLUSÕES}

1.O óleo essencial de $F$. vulgare tem atratividade significativa para fêmeas ápteras de A. gossypii;

2. Os óleos de $C$. citratus, $P$. aduncum, $C$. winterianus e azadirachtina são repelentes e reduzem a produção de ninfas desse pulgão;

\section{AGRADECIMENTOS}

Ao CNPq e FACEPE pela concessão de bolsa aos autores deste trabalho. A Solange Maria de França pelas sugestões nas análises estatísticas.

\section{REFERÊNCIAS}

ABRAMSON, C. I. et al. Effect of essential oil from citronella and alfazema on fennel aphids Hyadaphis foeniculi Passerini (Hemiptera: Aphididae) and its predator Cycloneda sanguinea L. (Coleoptera: Coccinelidae). American Journal of Environmental Science, v. 3, n. 1, p. 9-10, 2006.

BRITO, H. M. et al. Toxicidade de formulações de nim (Azadirachta indica A. Juss.) ao ácaro-rajado e a Euseius alatus De Leon e Phytoseiulus macropilis (Banks) (Acari: Phytoseiidae). Neotropical Entomology, v. 35, n. 4, p. 500-505, 2006.

COSIMI, S. et al. Bioactivity and qualitative analysis of some essential oils from Mediterranean plants against storedproduct pests: Evaluation of repellency against Sitophilus zeamais Motschulsky, Cryptolestes ferrugineus (Stephens) and Tenebrio molitor (L.). Journal of Stored Products Research, v. 45, n. 2, p. 125-132, 2009.

COSME, L. V.; CARVAlHO, G. A.; MOURA, A. P. Efeitos de inseticidas botânicos e sintéticos sobre ovos e larvas de Cycloneda sanguinea (Linnaeus) (Coleoptera: Coccinellidae) em condições de laboratório. Arquivos do Instituto Biológico, v. 74, n. 3, p. 251-258, 2007.
FAZOLIN, M. etal. Toxicidade do óleo de Piperaduncum L. a adultos de Cerotoma tingomarianus Bechyné (Coleoptera: Chrysomelidae). Neotropical Entomology, v. 34, n. 3, p. 485-489, 2005.

ISMAN, M. B. Botanical insecticides, deterrents, and repellents in modern agriculture and increasing regulated world. Annual Review of Entomology, v. 51, p. 45-66, 2006.

JUSTINIANO, W. et al. Eficiência do Óleo de Neem no Controle do Ácaro da Leprose dos Citros Brevipalpus phoenicis (Geijskes, 1939). Pesquisa Agropecuária Tropical, v. 39, n. 1, p. 38-42, 2009.

LIMA, R. K. et al. Composição de óleos essenciais de Anis-estrelado Illicium verum L. e de Capim-limão Cymbopogom citratus (DC.) Stapf.: Avaliação de Efeito Repelente sobre Brevicoryne brassicae (L.) (Hemiptera: Aphididae). BioAssay, v.3, p. 1-6, 2008.

LOVATTO, P. B.; GOETZE, M.; THOMÉ, G. C. H. Efeito de extratos de plantas silvestres da família Solanaceae sobre o controle de Brevicoryne brassicae em couve (Brassica oleraceae var. acephala). Ciência Rural, v. 34, n. 4, p. 9711-978, 2004.

MARTINEZ, S. S.; VAN ENDEM, H. F. Growth disruption, abnomalities and mortality of Spodoptera littoralis (Boisduval) (Lepidoptera: Noctuidae) caused by Azadirachtin. Neotropical Entomology, v. 30, n. 1, p. 113-124, 2001.

MARTINEZ, S. S. O nim Azadirachta indica: natureza, usos múltiplos, produção. 2. ed. Londrina: Instituto Agronômico do Paraná, 2002. 142 p.

MORDUE, A. J.; BLACKWELL, A. Azadirachtin: An update. Journal of Insect Physiology, v. 39, n. 11, p. 903-924, 1993.

NERIO, L. S.; OLIVERO-VERBEL, J.; STASHENKO, E. Repellent activity of essential oils from seven aromatic plants grown in Colombia against Sitophilus zeamais Motschulsky (Coleoptera). Journal of Stored Products Research, v. 45, n. 3, p. 212-214, 2009.

NERIO, L. S.; OLIVERO-VERBEL, J.; STASHENKO, E. Repellent activity of essential oils: a review. Biosource Technology, v. 101, n. 1, p. 372-378, 2010.

OBENG-OFORI, D. Plant oils as grain protectants against infestations of Cryptolestes pussilus and Rhyzopertha dominica in stored grain. Entomologia Experimentalis et Applicata, v. 77, n. 2, p. 133-139, 1995.

OLIVEIRA, J. E. M. et al. Desenvolvimento de metodologia de criação e multiplicação de Aphis gossypii: avanços e sucessos. Comunicata Scientiae, v. 1, n. 1, p. 65-68, 2010.

OLIVEIRA, J. V.; VENDRAMIM, J. D. Repelência de óleos essenciais e pós vegetais sobre adultos de Zabrotes subfasciatus (Boh.) (Coleoptera: Bruchidae) em sementes de feijoeiro. Anais da Sociedade Entomológica do Brasil, v. 28, n. 3, p. 549-555, 1999.

PAVELA, R.; VRCHOTOVÁ N.; BOZENA, S. Reppellency and toxicity of three Impatiens species (Balsaminaceae) extracts on Myzus persicae Sulzer (Homoptera: Aphididae). Journal of Biopesticides, v. 2, p. 48-51, 2009.

RAJENDRAN, S.; SRIRANJINI, V. Plant products as fumigants for stored-product insect control. Journal of Stored Product Research, v. 44, n. 2, p. 126-135, 2008. 
ROEL, A. R. et al. Efeito do extrato acetato de etila de Trichilia pallida Swartz (Meliaceae) no desenvolvimento e sobrevivência da lagarta-do-cartucho. Bragantia, v. 59, n. 1, p. 53-58, 2000.

ROEL, A. R. Utilização de plantas com propriedades inseticidas: uma contribuição para o Desenvolvimento Rural Sustentável. Revista Internacional de Desenvolvimento Local, v. 1, n. 2, p. 43-50, 2001.

SAS ISNTITUTE. SAS/STAT: User's guide, version 8.02, TS level 2MO. Cary: SAS Institute, 2001.

SCHMUTTERER, H. Properties and potential of natural pesticides from the neem tree, Azadirachta indica. Annual Review Entomology, v. 35, p. 271-297, 1990.

SILVA, F. A. C.; MARTINEZ, S. S. Effect on Neem Seed Oil Aqueous Solutions on Survival and Development of the Predator Cycloneda sanguinea (L.) (Coleoptera: Coccinellidae). Neotropical Entomology, v. 33, n. 6, p. 751-757, 2004.
TAVARES, M. A. G. C.; VENDRAMIM J. D. Bioatividade da Erva-de-Santa-Maria, Chenopodium ambrosioides L., sobre Sitophilus zeamais Mots. (Coleoptera: Curculionidae). Neotropical Entomology, v. 34, n. 2, p. 319-323, 2005.

VENDRAMIM, J. D.; CASTIGLIONI, E. Aleloquímicos, resistência de plantas e plantas inseticidas. In: GUEDES, J. C.; COSTA I. D.; CASTIGLIONI, E. Bases e técnicas do manejo de insetos. Santa Maria: UFSM/CCR/DFS, 2000. 128 p.

VIEIRA, P. C.; MAFEZOLI J.; BIAVATTI M. W. Inseticidas de origem vegetal. In: FERREIRA, J. T. B.; CORRÊA A. G.; VIEIRA, P. C. Produtos naturais no controle de insetos. São Carlos: EdUFSCar. 2001. 176 p.

WÄCKERS F. L.; VAN RIJN P. C. J.; BRUIN J. Plantprovided food for carnivorous insects. Cambridge University Press, 2007. 368 p. 